\title{
CONHECIMENTO ESPECÍFICO DE ENFERMEIROS DE UM HOSPITAL UNIVERSITÁRIO ACERCA DO CUIDADO AO IDOSO
}

Thiago Lago da Silva ${ }^{1}$, Silvana Sidney Costa Santos² ${ }^{2}$ Marlene Teda Pelzer ${ }^{3}$, Edison Luiz Devos Barlem ${ }^{4}$, Tatiane Alonso

Arrieche $^{5}$

RESUMO: O objetivo deste estudo foi identificar o conhecimento específico de enfermeiros de uma unidade de clínica médica de um hospital universitário do Sul do Brasil, acerca do cuidado à pessoa idosa. Foram sujeitos oito enfermeiras, com as quais se utilizou entrevista gravada e cujos depoimentos deram origem às categorias de análise, assim apresentadas: Pessoa Idosa; Política Nacional do Idoso; Preparo para Cuidar do Idoso; Cuidados Específicos de Enfermagem para o Idoso. Diante dos resultados, faz-se necessária a organização de um curso de aperfeiçoamento direcionado às enfermeiras investigadas, para que elas passem a ser multiplicadoras em relação à sua equipe e, assim, possam otimizar a qualidade de cuidados de enfermagem específicos prestado às pessoas idosas hospitalizadas.

PALAVRAS-CHAVE: Enfermagem; Cuidados de enfermagem; Idoso.

\section{THE SPECIFIC NURSING KNOWLEDGE REGARDING OLD PEOPLE CARING INA UNIVERSITY-HOSPITAL}

\begin{abstract}
The aim of this study was to identify the nurses' specific knowledge to take care of old people. It took place on a clinical unit of a university hospital from southern Brazil. Eight female nurses were interviewed. Their speeches originated the categories of analysis that follow: Old People; Old People's National Policy; Preparation to care of Old people; Nursing Specific care directed to old people. Results show that is necessary to organize an improvement course directed to the investigated nurses, then they will be multipliers in relation to their team and they will be able to improve the quality of the specific nursing care to old people in the hospital.
\end{abstract}

KEYWORDS: Nursing; Nursing care; Agred.

\section{CONOCIMIENTO ESPECÍFICO DE LOS ENFERMEROS DE UN HOSPITAL UNIVERSITARIO ACERCA DEL CUIDADO ALANCIANO}

RESUMEN: El objetivo de este estudio fue identificar el conocimiento específico de enfermeros en una unidad de clínica médica de un hospital universitario del Sur del Brasil, acerca del cuidado a la persona anciana. Fueran sujetos ocho enfermeras, con las cuales fue utilizada la entrevista gravada y cuyos testimonios condujeron a las categorías de análisis, así presentadas: Persona Anciana; Política Nacional del Anciano; Preparación para el Cuidado del Anciano; Cuidados Específicos de Enfermería para el Anciano. Delante de los resultados, se vuelve necesaria la organización de un curso de perfeccionamiento dirigido a las enfermeras investigadas, de manera que ellas conviértanse en multiplicadoras en relación a su equipo y así, puedan optimizar la calidad de los cuidados de enfermería específicos prestados a las personas ancianas hospitalizadas.

PALABRAS CLAVE: Enfermería; Atención de enfermería; Cuidado; Anciano

\footnotetext{
${ }^{1}$ Aluno. Departamento de Enfermagem. Universidade Federal do Rio Grande-UFRG.

${ }^{2}$ Enfermeira. Doutora. Professora. Departamento de Enfermagem-UFRG. Líder do Grupo de Estudo e Pesquisa em Gerontogeriatria, Enfermagem/Saúde e Educação (GEP-GERON).

${ }^{3}$ Enfermeira. Doutora. Professora. Departamento de Enfermagem-UFRG. Líder do GEP-GERON.

${ }^{4}$ Enfermeiro. Aluno do Mestrado em Enfermagem-UFRG. Professor do SENAC/Rio Grande/RS. Integrante do GEP-GERON.

${ }^{5}$ Enfermeira do Hospital Universitário da FURG. Aluna do Mestrado em Enfermagem-UFRG.
}

Autor correspondente:

Silvana Sidney Costa Santos

Rua Duque de Caxias, 197/503 - 96200-020 - Rio Grande-RS

Recebido: 13/08/08

E-mail: silvanasidney@terra.com.br.

Aprovado: 06/02/09 


\section{INTRODUÇÃO}

O Brasil organiza-se para responder às crescentes demandas de sua população que envelhece. A Política Nacional do Idoso, promulgada em 1994 e regulamentada em 1996, assegura direitos sociais à pessoa idosa, criando condições para promover sua autonomia, integração e participação efetiva na sociedade e reafirmando o direito à saúde nos diversos níveis de atendimento do SUS, por meio da Lei n. 8.842/ 94 e do Decreto n. 1.948/96 ${ }^{(1)}$.

A Política Nacional do Idoso (PNI) afirma que, por lei, é considerada idosa toda pessoa maior de 60 anos, a qual tem direitos garantidos. Em 2003, foi aprovado o Estatuto do Idoso, Lei n. 10.741, ampliando a resposta do Estado e da sociedade às necessidades da população idosa, porém essa aprovação não trouxe consigo meios para financiar as ações propostas. $\mathrm{O}$ Capítulo IV do Estatuto estabelece especificamente sobre o papel do SUS na garantia da atenção à saúde da pessoa idosa, de forma integral, em todos os níveis de atenção $^{(2)}$.

Em fevereiro de 2006 foram publicadas, por meio da Portaria n. 399/GM, as Diretrizes do Pacto pela Saúde, que apresenta três dimensões: Pacto em Defesa do SUS, Pacto pela Vida e Pacto de Gestão. O Pacto pela Vida ${ }^{(1)}$ reforça no SUS, o movimento da gestão pública por resultados. Nesse documento, a saúde do idoso aparece como uma das seis prioridades das três esferas de governo, sendo apresentada uma série de ações que visam à implementação de algumas das diretrizes da Política Nacional do Idoso.

As diretrizes no Pacto pela Vida a serem seguidas na saúde do idoso são: promoção do envelhecimento ativo e saudável; atenção integral à saúde do idoso; estímulo às ações intersetoriais, visando à integralidade da atenção; provimento de recursos capazes de assegurar a qualidade da atenção à saúde do idoso; estímulo à participação e fortalecimento do controle social; formação e educação permanente dos trabalhadores de saúde do SUS, na área de saúde do idoso; divulgação e informação sobre a Política Nacional de Saúde da Pessoa Idosa (PNSPI) para trabalhadores de saúde, gestores e usuários do SUS; promoção de cooperação nacional e internacional nas experiências de atenção à saúde dos idosos; apoio ao desenvolvimento de estudos e pesquisas ${ }^{(1)}$.

Ainda em relação à saúde do idoso, as ações estratégicas devem ser: aplicação da Caderneta de Saúde da Pessoa Idosa; conhecimento do Manual de
Atenção Básica e Saúde para a Pessoa Idosa; realização do Programa de Educação Permanente à Distância, para os trabalhadores que atuam na rede de atenção básica de saúde; realização do Acolhimento; Assistência Farmacêutica adequada; Atenção Diferenciada na internação, aplicando-se a avaliação geriátrica global realizada por equipe multidisciplinar a todo idoso hospitalizado; implantação da Atenção Domiciliar ${ }^{(1)}$.

Para que os enfermeiros desenvolvam, de forma adequada, cuidados ao ser humano idoso, alguns caminhos necessitam ser considerados ${ }^{(3: 89)}$, dentre os quais

[...] manutenção do bem-estar e vida autônoma, sempre que possível no ambiente domiciliar, onde tais cuidados centrem-se no idoso; desenvolvimento de um trabalho multidisciplinar e interdisciplinar, procurando partilhar responsabilidades, defendendo os direitos dos idosos/ família/comunidade; ampliação dos conhecimentos profissionais para além da área gerontogeriátrica, assumindo o ser humano como centro [...], direcionando o profissional da saúde e principalmente o da gerontogeriatria a oferecer uma melhoria na condição de vida do idoso, da sua família e da sua comunidade.

O processo de cuidar, em enfermagem gerontogeriátrica, consiste em olhar para a pessoa idosa, considerando os aspectos biopsicossociais e espirituais vivenciados pelo idoso e pela família, como clientes da enfermagem. Esse processo se dá em ações consecutivas de modo interativo, dialogal, entre quem provê o cuidado e quem o recebe. No cuidado à saúde de pessoas idosas, convencionam-se agrupar algumas metas principais, dentre elas: promoção de um viver saudável; compensação de limitações e incapacidades; provisão de apoio e controle no curso de envelhecimento; tratamento e cuidado específicos; facilitação do processo de cuidar ${ }^{(4)}$.

Essas metas direcionam o cuidado de enfermagem para o cuidado da vida e saúde, para a recuperação e a reabilitação do idoso no melhor grau possível privilegiando capacidades e habilidades de autocuidado, possibilitando-lhe continuar mantendo-se socialmente integrado, impedindo a perda da qualidade de vida e favorecendo sua manutenção ou melhoria ao longo da vida mediante a obtenção de suporte e acompanhamento ${ }^{(4)}$.

Ainda, no tratamento e cuidado específicos, a enfermagem presta cuidados ao idoso tão adequadamente quanto possível. Na medida de sua competência, tendo fundamento nos conhecimentos 
atualizados do campo da gerontogeriatria e na habilidade de aplicação das técnicas de tratamentos geriátricos específicos, particularmente das tecnologias cuidativas específicas de enfermagem e deve prover seus cuidados, favorecendo um processo de atendimento das reais necessidades do idoso sob cuidados e de seus respectivos familiares cuidadores ${ }^{(4)}$.

Percebe-se a necessidade de habilidades, competências e a responsabilidade que o enfermeiro precisa ter dentro das unidades de trabalho, desenvolvendo funções específicas e prestando cuidado aos idosos muitas vezes em situação grave. Assim, surgiu a seguinte questão de pesquisa: qual o conhecimento específico dos enfermeiros de uma unidade de clínica médica de um hospital universitário, acerca do cuidado ao idoso?

O presente estudo teve por objetivo identificar o conhecimento específico de enfermeiros de uma unidade de clínica médica de um hospital universitário do sul do país, acerca do cuidado à pessoa idosa.

\section{METODOLOGIA}

Trata-se de uma pesquisa exploratória e descritiva, com abordagem qualitativa, realizada em unidade de clínica médica de um hospital universitário que se encontra localizado no extremo sul do Brasil, no Estado do Rio Grande do Sul. A unidade de clínica médica tem 49 leitos, é constituída por 64 trabalhadores de enfermagem, sendo oito enfermeiras e 56 técnicos e auxiliares de enfermagem.

Foram sujeitos deste estudo as oito enfermeiras da unidade de clínica médica, que atuavam nos turnos manhã, tarde e noite, às quais foi aplicada uma entrevista gravada, durante seus plantões, em dias e horários agendados previamente, no período de março e abril de 2008, contendo dados de identificação como: sexo, idade, local de formação, tempo de formada, conteúdo sobre cuidado ao idoso no período acadêmico, realização de pós-graduação, tempo de atuação profissional e tempo de atuação na unidade de clínica médica. Os dados específicos tiveram como parâmetros: definição de pessoa idosa, conhecimento sobre a política para os idosos, preparo da equipe de enfermagem para cuidar de idosos internados, diferença entre cuidar de um idoso e de outras pessoas, os cuidados que poderiam ser específicos aos idosos, interesse em realizar um curso voltado ao cuidado da pessoa idosa, além de sugestões quanto ao cuidado ao idoso no local pesquisado.
Os preceitos da Resolução n. 196/96 do Conselho Nacional de Ética em Saúde para a Pesquisa com Seres Humanos foram observados. O projeto foi aprovado pelo Comitê de Ética da Santa Casa de Caridade do Rio Grande com número 690537/2007. As participantes foram informadas do objetivo e metodologia do estudo e, naquele momento, solicitadas a assinarem o Termo de Consentimento Livre e Esclarecido. Cada participante foi identificada como Enfermeira (E) e com um número correspondente à entrevista, sendo E1 a primeira enfermeira entrevistada, e assim por diante, até a enfermeira E8.

Após término da entrevista, os dados gravados foram transcritos. Cada participante, em outro momento, leu tudo o que foi anotado, a fim de reconhecer como autêntico o que havia sido respondido para, só então, os dados serem analisados. A análise se deu primeiro pela leitura flutuante das entrevistas, sem buscar, ainda, qualquer interpretação do que estava sendo exposto, ou sem qualquer tentativa de identificar qualquer atributo ou elemento ali contido. Segundo, pelas releituras tantas vezes quantas foram necessárias, com o objetivo de apreender os momentos significativos. Terceiro, pela reflexão acerca dos achados e sua a relação com bibliografias pertinentes, de modo a identificar o conhecimento específico da enfermeira que atua em unidade de clínica sobre cuidado ao idoso, procurando apreender esse conhecimento $^{(5)}$.

\section{RESULTADOS E DISCUSSÃO}

Os resultados são apresentados considerando a caracterização dos sujeitos investigados e as cinco categorias identificadas na análise dos dados: Pessoa Idosa; Política Nacional do Idoso; Preparo para Cuidar do Idoso; Cuidados Específicos de Enfermagem para o Idoso, apresentadas a seguir.

\section{Caracterização dos sujeitos}

As oito enfermeiras da unidade de clínica médica, dos turnos manhã, tarde e noite tinham idades entre 28 e 50 anos. Quatro enfermeiras se formaram pela Universidade Federal do Rio Grande (FURG), e quatro pela Universidade Federal de Pelotas (UFPel). O tempo de conclusão do curso de graduação variou de quatro a 26 anos. Quatro enfermeiras tiveram, durante sua formação, algum conteúdo sobre cuidado ao idoso, quando cursaram a disciplina de Saúde do 
Adulto. Três enfermeiras estavam cursando o mestrado em enfermagem. Seis enfermeiras tinham curso de especialização. O tempo de atuação na unidade de clínica médica variava de um ano e dois meses a dez anos.

\section{Pessoa Idosa}

Segundo convenções sociodemográficas atuais, são consideradas idosas todas as pessoas de mais de 60 anos, nos países em desenvolvimento, e de mais de 65 nos países desenvolvidos ${ }^{(6)}$. Essa convenção mostra que o envelhecimento e a condição de ser idoso estão diretamente relacionados à qualidade de vida que os países e seus governos oferecem aos habitantes.

O ser idoso é o resultado final de um conjunto envolvendo o envelhecimento, que é um processo, e a velhice, que compõe uma das fases da vida ${ }^{(7)}$. Para as enfermeiras investigadas, eram pessoas idosas aquelas com mais de 60 anos:

Pessoa com mais de 60 anos, [...] que pode possuir ou não alguma debilidade para andar ou para se comunicar $[\ldots]($ E.2).

[...] em primeiro lugar a faixa etária, a partir de 60 anos. [...] as limitações que eles têm (E.6).

[...] a partir dos 60 anos, [...] não sei [...] os parâmetros que definem (E.8).

As enfermeiras também consideraram idosas as pessoas doentes que, mesmo ainda não tendo 60 anos e mais, apresentassem comprometimento na saúde:

A idade é muito relativa, é mais a partir da saúdel doença [...] (E.4).

A afirmativa de E4, contraria a literatura pois envelhecer não é sinônimo de doença, embora, alguns idosos expressem uma síndrome de fragilidade, caracterizada por fraqueza muscular, fadiga e baixa tolerância aos estressores físicos e psicológicos, sinalizando condição de saúde vulnerável, que os coloca em risco de quedas, incapacidade e morte antecipada ${ }^{(8)}$.

Muitas vezes as doenças trazem fatores emocionais de regressão, aumentando assim a impotência, dependência e insegurança, causando sintomas de preocupações, angústias, medos, alterações na auto-imagem e algum nível de dependência ${ }^{(9)}$.
Entende-se o processo de envelhecimento como sendo um

[...] processo dinâmico e progressivo, no qual há perdas morfológicas, fisiológicas, bioquímicas e psicológicas, que determinam a perda progressiva da capacidade de adaptação do indivíduo ao meio ambiente, ocasionando maior vulnerabilidade e maior incidência de processos patológicos, que determinam por levá-lo à morte ${ }^{(10: 3)}$.

O envelhecimento apresenta-se ainda como um processo universal e individual, uma experiência heterogênea e subjetiva, refletindo fenômenos fisiológicos, de comportamento social, ou ainda cronológicos, isto é, relacionados à idade. No seu desenvolvimento, ocorrem mudanças, podendo ou não estar inter-relacionadas, que são biológicas e psicossociais $^{(9)}$.

\section{Política Nacional do Idoso}

A Política Nacional do Idoso é uma diretriz inovadora, com poder político-programático e de gestão de recursos humanos. Inserida na Política Nacional do Idoso está a Política Nacional de Saúde da Pessoa Idosa (PNSPI), que diz respeito, mais diretamente, aos trabalhadores da saúde, e especificamente aos enfermeiros.

As enfermeiras entrevistadas relataram:

\section{[...] tem várias políticas. [...] Estatuto do Idoso} que [diz] em relação aos cuidadores, que a família tem que dar um amparo para o idoso [...], em relação ao lazer [...], transporte [...], saúde [...], tem auxílio benefício, que eles têm direito, e muitas pessoas não sabem (E.7).

Percebe-se que E7 tem algum conhecimento acerca da Política Nacional do Idoso, quando cita algumas questões relacionadas ao Estatuto do Idoso. Importante que as enfermeiras conheçam a Política Nacional de Saúde da Pessoa Idosa (PNSPI), cujas finalidades são recuperar, manter e promover a autonomia e a independência dos idosos, direcionando medidas coletivas e individuais de saúde para esse fim, em consonância com os princípios e diretrizes do SUS. É alvo dessa política todo cidadão e cidadã brasileiros com 60 anos ou mais ${ }^{(11)}$ e, na sua gênese, objetiva-se a melhor qualidade de vida possível. Esse é um conhecimento necessário a todo enfermeiro que atua em unidade de clínica médica, considerando que muitos 
dos doentes internados são pessoas idosas. Porém, alguns enfermeiros ainda não despertaram para tal tema:

[...] tenho pouco conhecimento [...] direito ao acompanhante, da prioridade em instituições de saúde, que teria que ter prioridade nas filas em supermercado, teatros, direito ao passe livre (E.8).

Quando o enfermeiro despertar para a devida importância da Política Nacional do Idoso, tornar-seá mais fácil defender os direitos dos cidadãos idosos e haverá (re)conhecimento das necessidades específicas desses clientes. Esse sentimento foi expressado nas enfermeiras pesquisadas:

Sim [é importante], porque o idoso é um cliente diferenciado [...], nas suas necessidades humanas básicas (E.2).

[...] é importante, [...] poder trazer uma série de benefícios, [...] alguns idosos têm necessidades especiais, dificuldades financeiras, não acesso à saúde, ao lazer [...] (E.8).

[...] as pessoas idosas não são respeitadas pelos familiares. Uma vez que as necessidades delas ficam afetadas e elas ficam dependentes, a maioria dos familiares some e o idoso fica [no hospital] sozinho, sem assistência familiar [...] (E.6).

Conforme o depoimento de E6, a família apresenta importante papel junto ao idoso. Grande parte dos idosos vive com a família. O idoso dependente passará a precisar de ajuda para realizar sua higiene pessoal, vestir-se, alimentar-se, além de poder haver a perda da continência urinária e fecal. E, quando acamado, necessitará de cuidados especiais para a prevenção de contraturas, encurtamentos, dores e desenvolvimento de escaras ${ }^{(12)}$.

\section{Preparo para Cuidar do Idoso}

Ao considerar o idoso em sua singularidade e especificidade, ficam evidentes a importância e necessidade de uma atenção especializada e diferenciada, ressaltando-se a urgência da capacitação dos trabalhadores da enfermagem, por meio de programas específicos para o cuidado ao idoso.

Quando se tem conhecimento sobre cuidar da pessoa idosa durante a formação acadêmica, têm-se melhores oportunidades de prestar um cuidado mais adequado ao idoso. Os profissionais que tiveram preparo no cuidado ao idoso e receberam algum tipo de iniciação em princípios de clínica do idoso adquiriram segurança para realizar um cuidado mais especifico, tornando-se instrumentalizados para tal ação ${ }^{(13)}$. Foram opiniões das enfermeiras pesquisadas:

[...] aprendi um pouco na [atividade prática de] clínica médica. Está faltando um conhecimento mais aprofundado sobre os cuidados [...] em relação ao idoso, porque a gente cuida de uma maneira geral, (...) poderíamos fazer muito mais [...] (E.1).

Tive em saúde do adulto [...] não, como eu gostaria [...] (E.2).

Muito vago, vi alguma coisa sim [...]. Não tive separado um curso voltado para a pessoa idosa (E.4).

Os depoimentos mostram que as enfermeiras tiveram pouco contato com conteúdos/temas sobre cuidado ao idoso, tal conhecimento precisa ser mais consolidado, elas necessitariam terem avançado durante a prática profissional, já que a maior parte de seus clientes são os maiores de 60 anos. Necessita-se investigar a presença de outros agravantes ao bemestar e assim, incorporar novos conhecimentos à prática de enfermagem, quando cuidamos de pessoas idosas ${ }^{(14)}$.

\section{Cuidados específicos de enfermagem para o idoso}

O cuidar envolve um agir e uma atitude da enfermeira integrados por duas formações: a pessoal e a profissional. As possíveis repercussões desses valores, com reflexos na prática dos enfermeiros, podem ser percebidas no cotidiano, no relacionamento entre clientes e trabalhadores de enfermagem. Esse relacionamento perpassa pela subjetividade do profissional que assiste, intervindo no cuidar e no agir humanos ${ }^{(15)}$.

Como a população idosa brasileira é a que mais cresce, proporcionalmente, no país, passa-se a refletir que tais pessoas carecem de cuidados específicos e multidimensionais. O cuidado direcionado ao idoso é uma ação complexa e interdisciplinar, por isso a complexidade dos problemas de saúde dos idosos necessita ser mais bem trabalhada, quando se acorre de auxílio do desenvolvimento dessa ação ${ }^{(3)}$. É preciso um conjunto de trabalhadores para atender adequadamente às necessidades de uma pessoa idosa, 
pois um só profissional, oriundo de uma só disciplina, não dará conta desse atendimento, que deve ser integral.

Necessita-se integrar a enfermagem com a ação do cuidar, e entendê-la, não como uma prática reducionista na ação curativa e limitada, mas fundamentada na percepção do ser humano, o idoso, como pessoa com seus valores, crenças e experiências ${ }^{(15)}$. Um bom trabalho disciplinar alavanca um trabalho prático na equipe multidisciplinar, correspondendo à maior riqueza da geriatria e da gerontologia, que é a interdisciplinaridade.

Verifica-se essa percepção, nos depoimentos das enfermeiras:

A pessoa idosa requer alguns cuidados diferenciados, as limitações ficam mais presentes, a promoção do autocuidado [é] muito importante, [cuidado] com a pele e da parte mental (E.1).

O idoso, como todos os pacientes, merece carinho $e$ atenção. [...] temos que ter um cuidado especial para lidar com eles (E.3).

Ter cuidado até mesmo no material que se usa, porque a pele é sensível; os sinais vitais são diferentes [...] e eles usam muitas medicações (E.4).

[...] o idoso tem suas especificidades [...] é mais fragilizado, temos que ter mais cuidado com eles, paciência, e sabermos o que é realmente uma patologia ou o que é da idade mesmo, então temos que estudar e ler (E.7).

O cuidar é uma atividade que vai além do atendimento às necessidades básicas do ser humano, no momento em que ele está fragilizado. $\mathrm{O}$ cuidado fornece o elo de passagem da transcendência para a imanência e da história para a utopia, ou seja, é por meio do cuidado que o espírito - transcendente, dá forma ao corpo - imanente ${ }^{(16)}$.

Não cuidar do idoso como se ele fosse [...] inútil, [...] cuidar da pele, mudanças de decúbito, [...] alimentação, [...] se estiver lúcido, orientado [...] estimular o autocuidado [...] (E.1).

[...] não é cuidado técnico, mas é respeito [...] carinho, [cuidado] com a formação de escaras. [...] Cuidado com as técnicas de punções. Auxílio para ir ao banheiro, para evitar quedas e fraturas (E.3).
Cuidado na maneira de pegar; ajudar a caminhar; a descer ou movimentação no leito. [...] cuidados com os idosos demenciados (E.4).

[...] colocar em colchão piramidal, mudar de decúbito a cada duas horas (E.6).

Dar o banho e passar óleo para prevenir as escaras (E.7).

Ao se avaliar uma pessoa idosa de forma mais integral, têm-se mais possibilidades de atender as suas necessidades primárias básicas afetadas. Outra preocupação das enfermeiras diz respeito à avaliação funcional, conforme depoimento:

\section{[...] [há] necessidade de avaliação específica (E.2).}

Essa avaliação específica, referida por E2, diz respeito à avaliação funcional do idoso, que faz parte do cuidado de enfermagem, com ênfase na pessoa e nos sistemas de apoio com os quais ela pode contar, pois o ser humano idoso deve ser assistido de maneira individualizada, levando em consideração suas limitações físicas, psíquicas e ambientais ${ }^{(17)}$.

Em Gerontologia, ciência que estuda o envelhecimento do ser humano, a avaliação funcional é de extrema importância para promover a reabilitação, sendo o desempenho nas atividades da vida diária o parâmetro aceito e reconhecido para realizá-la.

A avaliação da capacidade funcional dos idosos permite ao enfermeiro e aos demais membros da equipe multidisciplinar uma visão mais precisa quanto à severidade da doença, assim como quanto ao impacto da comorbidade ${ }^{(17)}$.

A avaliação funcional é uma forma de estimar a saúde de um idoso, tendo como base a capacidade do idoso em realizar as Atividades de Vida Diária (AVD), determinando assim, o seu grau de independência e autonomia. Enquanto que a impossibilidade de realização de determinado ato caracteriza uma condição incapacitante, a capacidade significaria a realização de um ato.

Torna-se essencial a avaliação funcional no cuidado ao idoso, porque oferece uma abordagem sistemática para avaliar déficits que geralmente passam despercebidos. Ela ajuda o profissional a identificar problemas e utilizar medidas adequadas, como determinar o tipo de tratamento; proporciona meios para avaliar o progresso e a deterioração com o passar do 
tempo; e auxilia o trabalhador na avaliação da capacidade do idoso para viver sozinho, em segurança ${ }^{(18)}$. Essa avaliação torna-se, a cada dia, uma das principais ferramentas de cuidado multidisciplinar da pessoa idosa, sendo necessária sua aprendizagem pelos enfermeiros.

\section{CONSIDERAÇÕES FINAIS}

Este estudo nos permitiu avaliar o conhecimento de enfermeiras de uma unidade de clínica médica de um hospital universitário, acerca do cuidado ao idoso. Foi possível constatar que essas trabalhadoras desenvolviam, junto ao idoso, um cuidado mais geral, e que havia um déficit de conhecimento quanto à Política Nacional do Idoso e, principalmente, quanto ao cuidado específico à pessoa idosa.

Em muitas situações, o cuidado desenvolvido pela equipe de enfermagem parece distanciar a relação teoria-prática, que se afigura como algo aleatório. As enfermeiras da unidade afirmaram que os cuidados prestados não tinham como enfoque principal o atendimento exclusivo ao idoso, ou seja, não havia condições de cuidar levando-se em consideração a faixa etária, mas, sim, o processo de adoecimento.

Houve interesse das enfermeiras pesquisadas em participar de um curso acerca do cuidado específico à pessoa idosa, desde que realizado junto à equipe de enfermagem. Quanto a essa questão, posteriormente será contatado o Serviço de Educação Continuada do hospital universitário, para elaboração de uma proposta conjunta.

Acredita-se ser necessário que os enfermeiros se conscientizem da importância da avaliação direcionada ao idoso, para darem maior credibilidade e cientificidade ao cuidado prestado. A avaliação realizada pelo enfermeiro, se fundamentada por conhecimentos científicos, passa a ser mais efetiva, pois, através dela, o enfermeiro é capaz de identificar o que é prioritário para o idoso, em suas necessidades gerais. Para tanto, é preciso conhecimento específico por parte do enfermeiro, reforçando a questão da educação permanente como uma ação constante.

À guisa de conclusão, considera-se como fundamental observar a figura humana do idoso sob a ótica de uma contextualização que contemple sua diversidade e individualidade, também quando estiver em uma situação eventual de hospitalização. A enfermagem brasileira, diante do acelerado envelhecimento populacional, deve pautar sua prática assistencial na apreensão ampliada das necessidades de saúde do usuário idoso.

\section{REFERÊNCIAS}

1. Ministério da Saúde (BR). Pacto pela Saúde, 2006. Portaria n 399 - MS/ GM - 22 de fevereiro de 2006 Diretrizes Operacionais do Pacto pela Saúde. Brasília; 2006.

2. Ministério da Saúde(BR). Lei n. ${ }^{\circ} 10.741$, de $1^{\circ}$ de outubro de 2003. Estatuto do Idoso. Brasília; 2003.

3. Santos SSC. O desenvolvimento sustentável e o cuidado ao idoso: desafios convergentes. In: Cianciarullo TI, Cornetta VK, organizadores. Saúde, desenvolvimento e globalização - um desafio para os gestores do terceiro milênio. São Paulo: Ícone; 2002. p. 87-100.

4. Gonçalves LHT, Alvarez AM. O cuidado na enfermagem gerontogeriátrica: conceito e prática. In: Freitas EV, Py L, Cançado FAX, Doll J, Gorzoni ML, organizadores. Tratado de geriatria e gerontologia. $2^{\mathrm{a}}$ ed. Rio de Janeiro: Guanabara Koogan; 2006. p. 1114-5.

5. Martins J, Bicudo MAV. A pesquisa qualitativa em psicologia: fundamentos e recursos básicos. $6^{\mathrm{a}}$ ed. São Paulo: Morais; 2000.

6. Neri AL. Idosos, velhice e envelhecimento. In: Neri AL. Palavras chave em gerontologia. $2^{\text {a }}$ ed. Campinas: Alínea; 2005. p. 114-5.

7. Papaleo Netto M. O estudo da velhice: histórico, definição do campo e termos básicos. In: Freitas EV, Py L, Cançado FAX, Doll J, Gorzoni ML, organizadores. Tratado de geriatria e gerontologia. $2^{\mathrm{a}}$ ed. Rio de Janeiro: Guanabara Koogan; 2006. p. 2-12.

8. Espinoza S, Walston J. Frailty in older adults: insigths and interventions. Cleveland Clin. J. Medicine. $2005 \mathrm{Jan} /$ Feb;7(12):1105-12.

9. Diogo MJD, Duarte YAO. Cuidados em domicílio: conceitos e práticas. In: Freitas EV, Py L, Cançado FAX, Doll J, Gorzoni ML, organizadores. Tratado de geriatria e gerontologia. $2^{a}$ ed. Rio de Janeiro: Guanabara Koogan; 2006. p.1122-30.

10. Carvalho Filho ETD, Papaléo Netto M, Garcia YM. Biologia e teorias do envelhecimento. In: Carvalho Filho ETD, Papaléo Netto M. Geriatria fundamentos, clínica e terapêutica. 2 a ed. São Paulo: Atherneu; 2006. p.3.

11. Ministério da Saúde (BR). Portaria n. 2.528, de 19 de 
Outubro de 2006 - Política Nacional de Saúde da Pessoa Idosa. Brasília; 2006b.

12. Melo MA, Driusso P. Proposta fisioterapêutica para os cuidados de portadores da doença de Alzheimer. Envelhecimento e Saúde. 2006;12(4):11-8.

13. Silva BT, Santos, SSC. Avaliação do ensino da disciplina Enfermagem Gerontogeriátrica do curso de graduação em enfermagem da FURG. Cogitare Enferm. 2007 Jan/ Mar;12(1):82-8.

14. Santana RF, Santos I. Como tornar-se idoso: um modelo de cuidar em enfermagem gerontológica. Texto Contexto Enferm. 2005 Jan/Mar;14(2):268-73.

15. Brum AKR, Tocantins FR, Silva TJES. O enfermeiro como instrumento de ação no cuidar do idoso. Rev Latino-Am Enferm. 2005 Nov/Dez;13(6):1019-26.

16. Caldas CP. Aspectos éticos: considerando as necessidades da pessoa idosa. In: Saldanha AL, Caldas CP, organizadores. Saúde do idoso a arte do cuidar. $2^{\mathrm{a}}$ ed. Rio de Janeiro: Interciência; 2004. p. 37-40.

17. Diogo MJD. O papel da enfermeira na reabilitação do idoso. Rev Latino-Am Enfermagem 2000 Jan;8(1):75-81.

18. Bowling A. La medida de la salud. Revisión de las escalas de medida de la calidad de vida. Espanha: Massan; 1994. 